\title{
Les bouleversements de la certification dans le secondaire
}

\section{Le cas de la Pologne}

The upheavals of certification in the secondary school. The case of Poland Los grandes cambios en la certificación en la enseñanza secundaria. El caso de Polonia

\section{Jan Kuriata}

\section{OpenEdition}

\section{Journals}

Édition électronique

URL : https://journals.openedition.org/ries/1368

DOI : 10.4000/ries. 1368

ISSN : 2261-4265

Éditeur

France Education international

Édition imprimée

Date de publication : 1 décembre 2004

Pagination : 29-33

ISBN : 978-2-85420-564-0

ISSN : $1254-4590$

Référence électronique

Jan Kuriata, «Les bouleversements de la certification dans le secondaire », Revue internationale d'éducation de Sèvres [En ligne], 37 | décembre 2004, mis en ligne le 17 novembre 2011, consulté le 06 juillet 2021. URL : http://journals.openedition.org/ries/1368 ; DOI : https://doi.org/10.4000/ries.1368 


\section{Les bouleversements de la certification dans le secondaire}

\section{Le cas de la Pologne}

\section{Jan Kuriata}

Plus de six mois après l'intégration de la Pologne dans l'Union européenne, arrive le moment où les débats souvent vifs ${ }^{1}$ concernant la matura, le baccalauréat polonais, parviennent à leur fin, suite à la décision prise par le ministère de l'Éducation nationale et du Sport polonais (MENiS). Dès l'année 2005, les élèves polonais n'auront plus qu'un seul système appelé «nowa matura» (nouveau baccalauréat).

\section{LA RÉFORME DE L'ENSEIGNEMENT SECONDAIRE POLONAIS}

La réforme de l'enseignement secondaire polonais a débuté en 1999 et a connu de nombreux avatars. La réduction de la durée de la scolarisation primaire, ramenée à six années (de six ou sept ans jusqu'à l'âge de douze ans) a laissé la place à la création d'un premier cycle du secondaire. Le premier cycle $\mathrm{du}$ secondaire prend place dans un établissement baptisé gimnzajum, plus ou moins proche du collège français. Ce cycle se termine par un examen sur lequel nous reviendrons plus loin. Le lycée d'enseignement général garde le nom de liceum. La scolarité y est ramenée à trois années. Les autres établissements prennent le nom de leur spécialité professionnelle, mais ils seront prochainement remplacés par un seul établissement, le liceum porofilowane, probablement à l'horizon de 2006. Le technikum devrait voir ses études réduites à trois années menant à la matura comme dans le liceum, suivies d'une année supplémentaire d'études professionnelles aboutissant à un examen et un diplôme professionnels.

\section{ORganisation}

Le système éducatif polonais a hérité de plusieurs systèmes eux-mêmes issus des différents régimes politiques qu'a connus le pays, notamment du système soviétique. Ces systèmes se juxtaposent parfois au prix de nombreux compromis et dérogations. Ainsi, le primaire dure six années, mais avec la

1. "La réforme de la matura en Pologne», Gauthier P-L., Kuriata J., Revue internationale d'éducation de Sèvres $\mathrm{n}^{\circ} 32,2003$. 
possibilité de scolariser les enfants dès l'âge de six ans dans des classes "zéro », opportunité que saisissent $90 \%$ des familles.

C'est donc au bout de sept années que la majorité des élèves accèdent au secondaire après avoir réussi un test «de compétence». Ce test est obligatoire à la fin de l'école primaire mais son rôle se limite à une évaluation formative sans avoir de caractère certificatif ni sommatif. Les élèves qui n’ont pas réussi à obtenir le minimum de points prévus sont malgré tout admis au collège si leurs notes finales se situent entre 2 et 6 alors que la grille d'évaluation se situe entre 0 et 6 .

Les études au collège durent trois années et les classes sont appelées : première, deuxième et troisième alors qu'à l'école primaire les enfants fréquentaient également la première, la deuxième ... jusqu'à la sixième. L'exercice de numération devient délicat!

Les collégiens, à la fin de leurs études comme à la fin du primaire, passent un test «de compétence» qui porte sur des éléments de langue polonaise, mathématiques, histoire, géographie ou sciences naturelles... Ce test, comme celui de l'école primaire, n'a pas de caractère certificatif ni sommatif mais les résultats sont pris en considération par les lycées au moment du recrutement et décident pratiquement de l'admission. Ainsi, certains élèves peuvent se voir refusés par tel ou tel lycée si le nombre de points obtenus au test n'est pas assez élevé; ceci est particulièrement vrai pour les lycées qui disposent de plus de candidats que de places disponibles.

Le test de compétence joue le rôle d'un examen final sans l'être réellement car les directions des lycées, outre les résultats des tests, sont assez libres dans le choix des épreuves et des concours à prendre en considération et qui peuvent augmenter le nombre de points des candidats. Sont ainsi pris en considération les résultats obtenus aux différents concours spécialisés par matières, comme les olympiades de mathématiques par exemple.

Il convient de souligner que les deux tests sont des examens «externes» car leurs contenus sont élaborés par une commission indépendante des écoles et leurs corrections réalisées par les experts indépendants. Nous apporterons plus de précisions concernant les commissions et les experts dans la suite du présent article.

Les études secondaires de ce qu'on pourrait appeler «le deuxième cycle» durent trois années. Les filières principales sont au nombre de cinq :

- le lycée général (liceum ogolnoksztalcace) et le lycée technique (liceum techniczne) : ces deux établissements comportent les classes I à III (seize à dixneuf ans). En matière de certification, une attestation de scolarité mentionne les disciplines et les notes obtenues lors de la dernière année. Il n’y a pas d'examen final. 
- le lycée professionnel (liceum zawodowe) comporte les classes I à III (seize à dix-neuf ans) et prévoit pour la certification la mention des notes obtenues et un examen de sortie (egzamin $z$ nauki zawodu);

- le technikum, classes I à IV (seize à vint ans), comporte également une attestation mentionnant les résultats de la dernière année et les résultats obtenus à l'examen de sortie (egzamin z przygotowania zawodowego) qui confère le titre de technicien.

- l'école professionnelle de base (szkola zasadnicza) forme en trois ans (de seize à dix-neuf ans) des ouvriers qualifiés dans différents secteurs de la production.

Les trois premiers établissements donnent accès au baccalauréat, la matura. On voit bien que les filières techniques et professionnelles ont peu à peu trouvé une voie vers la matura.

\section{LA NOWA MATURA : UNE EXPERTISE EXTERNE?}

L'examen terminal de l'enseignement secondaire, la matura, subit évidemment les contrecoups de cette réforme. La principale innovation est que la nouvelle matura devient un examen externe. En fait, avec cet art du compromis où excellent les autorités polonaises, l'examen revêt encore deux formes :

- une procédure interne est gérée par les établissements sans intervention de l'extérieur; elle consiste en des épreuves orales concernant la langue polonaise et une langue vivante (y compris les langues des minorités ethniques du pays).

- une procédure externe est mise en œuvre par un organisme nommé "Centralna Komisja Egzaminacyjna», qu'on peut traduire par «commission centrale d'examen» (CCE), qui est un organisme unique et centralisé pour toute la Pologne. Cette commission est structurée dans les régions en «commissions régionales d'examen» (CRE) au nombre de huit pour tout le pays, donc inférieur au nombre de voiévodies ${ }^{2}$. La partie externe de l'examen se compose d'épreuves écrites au niveau «basique» et au niveau «élargi».

Le niveau basique comprend la langue polonaise, une langue vivante (anglais, allemand, russe, français, espagnol) - la même qui était choisie en partie orale -, une épreuve de mathématiques, une matière à option (biologie, chimie, physique, astronomie, géographie, histoire, informatique, langue vivante supplémentaire, latin, grec, éducation civique, danses, histoire de l'art, mathématiques). Les élèves sont obligés de choisir la langue polonaise et une langue vivante ainsi qu'une matière parmi celles que nous venons de citer.

2. Circonscriptions administratives comparables aux régions en France. Il existe seize voiévodies en Pologne. 
Les matières proposées au niveau «élargi» sont uniquement les suivantes : biologie, physique et astronomie, histoire, histoire de la musique, histoire de l'art, langue grecque et culture antique, langue latine et culture antique, langue vivante, éducation civique, langue d'une minorité ethnique, savoir sur les danses, chimie, géographie, informatique, mathématiques. Les élèves peuvent choisir une, deux ou trois matières afin de pouvoir préparer une intervention au cours des épreuves de matura. Les élèves la préparent seuls mais ils peuvent réclamer quelques aides de la part des enseignants de leurs écoles, l'objectif étant d'élaborer un document prouvant le degré d'autonomie de l'élève.

La CCE et les CRE sont des organismes indépendants chargés de préparer et gérer les examens pour l'ensemble de l'enseignement primaire et secondaire. L'objectif est d'évaluer de manière indépendante et objective la qualité de l'enseignement et d'estimer les niveaux exigibles pour les passages du primaire au gimnzajun, puis au secondaire "deuxième cycle» (Le «deuxième cycle» est pourtant absent dans la nomenclature officielle du MENiS).

Actuellement, les membres des commissions sont désignés et en cours de formation. Ils ont recruté et formé dans chaque région des «leaders» pour chaque discipline, chargés à leur tour de former les enseignants à ces nouvelles procédures. Parallèlement sont actuellement formés des experts ou examinateurs dont la compétence sera validée par le ministère de l'Éducation nationale et du Sport (MENiS) en qualité de membres des jurys d'examen.

La matura ainsi prise en charge par les commissions nationales et régionales a donc radicalement changé de visage. Les questions, les sujets d'examen, les règlements sont préparés désormais à ce niveau en privant de ces compétences les enseignants des écoles secondaires responsables jusqu'à présent à la fois de la formation et de la certification! On peut facilement deviner que ce bouleversement a profondément heurté la plupart des enseignants habitués à ne pas être soumis aux évaluations extérieures.

L'échelle de notation est remplacée par un système de points à acquérir (crédits). C'est un changement fondamental qui bouscule la mentalité traditionnelle polonaise attachée aux anciens examens de maturité. Il est difficile de prévoir les réactions des élèves et de leurs parents lorsqu'ils connaîtront les résultats des épreuves organisées «autrement». La structure de la nouvelle matura occupe une grande place dans les médias en Pologne qui s'efforcent d'expliquer aux parents le nouveau système, système qui semble être bien compliqué pour des personnes étrangères au système éducatif polonais.

\section{LA NOWA MATURA ET LES UNIVERSITÉS}

La précision et la rigueur qui semblent présider à cette mise en place de la nouvelle matura incitent les universités à inscrire directement les titulaires 
de la matura sur les listes d'étudiants. Mais il est difficile de préciser quelle sera l'attitude ou plutôt les attitudes que vont adopter les établissements supérieurs face à la nouvelle matura. L'ensemble des établissements supérieurs n'a pas encore élaboré une position officielle claire et précise. Vu le statut des universités en Pologne, aucune loi ne peut les obliger à adopter une attitude ou une autre par rapport à la matura qui reste toujours un examen «non-universitaire» bien qu'il soit externe à l'école secondaire. Les universités sont censées ne pas organiser des examens comportant des matières présentes dans la nouvelle matura. Dans les cas où les examens universitaires ne comprendraient que des matières présentes à la matura, le classement des candidats se ferait alors selon le nombre de points acquis à la nouvelle matura.

La mise en marche du nouveau système des examens clôturant la formation secondaire en Pologne est un fait. Nous devons souligner la longueur $\mathrm{du}$ processus d'élaboration de la nouvelle matura par rapport aux projets d'origine. Les obstacles étaient multiples et de diverses origines. Tout d'abord, pour le milieu enseignant les examens «externes» bouleversaient les habitudes professionnelles existant en Pologne «depuis toujours». Les partis politiques et le syndicat des enseignants polonais (le ZNP) craignaient de perdre ainsi leur clientèle qui ne souhaitait pas de changements. Les parents craignaient des difficultés dans l'organisation des examens et surtout, on prévoit des problèmes dans l'accès aux établissements supérieurs. Ces craintes n’ont pas disparu car le printemps 2005 est devant nous et il est difficile d'estimer si la bataille est gagnée... 